\title{
Bone morphogenetic protein 4 regulates the budding site and elongation of the mouse ureter
}

\author{
Yoichi Miyazaki, ${ }^{1}$ Keisuke Oshima, ${ }^{1}$ Agnes Fogo, ${ }^{1,2}$ Brigid L.M. Hogan, ${ }^{3}$ \\ and Iekuni Ichikawa ${ }^{1,4}$ \\ ${ }^{1}$ Department of Pediatrics, \\ ${ }^{2}$ Department of Pathology, \\ ${ }^{3}$ Department of Cell Biology, and \\ ${ }^{4}$ Department of Medicine, Vanderbilt University Medical Center, Nashville, Tennessee 37232, USA
}

Address correspondence to: Iekuni Ichikawa, Vanderbilt University Medical Center, MCN C4204, 21st and Garland Avenue, Nashville, Tennessee 37232-2584, USA. Phone: (615) 322-7931; Fax: (615) 322-7929;

E-mail: iekuni.ichikawa@mcmail.vanderbilt.edu.

Received for publication August 25, 1999, and accepted in revised form February 7, 2000.

In the normal mouse embryo, $B m p 4$ is expressed in mesenchymal cells surrounding the Wolffian duct (WD) and ureter stalk, whereas bone morphogenetic protein (BMP) type I receptor genes are transcribed either ubiquitously (Alk3) or exclusively in the WD and ureter epithelium (Alk6). Bmp4 heterozygous null mutant mice display, with high penetrance, abnormalities that mimic human congenital anomalies of the kidney and urinary tract (CAKUT), including hypo/dysplastic kidneys, hydroureter, ectopic ureterovesical (UV) junction, and double collecting system. Analysis of mutant embryos suggests that the kidney hypo/dysplasia results from reduced branching of the ureter, whereas the ectopic UV junction and double collecting system are due to ectopic ureteral budding from the WD and accessory budding from the main ureter, respectively. In the cultured metanephros deprived of sulfated glycosaminoglycans (S-GAGs), BMP4-loaded beads partially rescue growth and elongation of the ureter. By contrast, when S-GAGs synthesis is not inhibited, BMP4 beads inhibit ureter branching and expression of Wnt 11, a target of glial cell-derived neurotrophic factor signaling. Thus, $B m p 4$ has 2 functions in the early morphogenesis of the kidney and urinary tract. One is to inhibit ectopic budding from the WD or the ureter stalk by antagonizing inductive signals from the metanephric mesenchyme to the illegitimate sites on the WD. The other is to promote the elongation of the branching ureter within the metanephros, thereby promoting kidney morphogenesis.

J. Clin. Invest. 105:863-873 (2000).

\section{Introduction}

Bone morphogenetic protein 4 (BMP4), a member of the TGF- $\beta$ superfamily of secretory signaling molecules, has been implicated in many aspects of embryonic development, ranging from establishment of the basic embryonic body plan to morphogenesis of individual organs, by regulating cell proliferation, differentiation, apoptosis, and cell fate determination (1). The essential role of BMP4 in embryonic development is confirmed by the lethality between E6.5 and 10.0 of mouse embryos homozygous for null mutations in Bmp4 $(2,3)$. Moreover, even in heterozygous mutants, several defects have been described, including skeletal abnormalities, eye defects, and cystic kidneys accompanied by urinary tract anomalies (4). This latter phenotype, in conjunction with the expression pattern of Bmp4 during the development of the excretory system (5), suggests that $B m p 4$ may have an important role in kidney and urinary tract organogenesis. In the present study, we describe defects in heterozygous $B m p 4$ null mutant mice $\left(B m p 4^{+/-}\right)$that mimic human congenital anomalies of the kidney and urinary tract (CAKUT) in many respects (6). Our in vivo and in vitro studies in mutant and wild-type mice identify some key ontogenic events of the kidney and urinary tract that are governed by BMP4.

\section{Methods}

Mice. The generation of Bmp4 heterozygous null mutant mice (Bmp4lacZneo $)$ has been described previously (3). Briefly, a TL1 embryonic stem cell line (derived from 129/SvEvTacfBR embryos; Taconic Farms, Germantown, New York, USA) that carries targeted Bmp4laczneo allele was injected into C57BL/6NHsd (Sprague Dawley; Harlan Bioproducts for Science Inc., Indianapolis, Indiana, USA) blastocysts; resulting male chimeras were mated with outbred Black Swiss

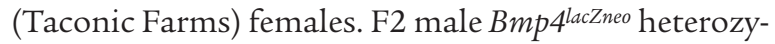
gotes on the genetic background of $129 / \mathrm{SvEv} \times$ Black Swiss were then serially backcrossed onto C57BL/6 females (The Jackson Laboratory, Bar Harbor, Maine, USA). Heterozygous $\left(\mathrm{Bmp}^{\text {lacZneo+/-}}\right)$ and wild-type $\left(^{+/+}\right)$ offspring between N2 and N4 generations were used in the present study. The Bmp4 laczneo genotype was determined by PCR analysis for the neo gene with the following primer sequences: neo1, 5'-TCCTGCCGAGAAAGTATCCATCAT-3', and neo 2, 5'-GTAAAGCACGAGGAAGCGGTCAGC-3’. 
Histology. At least 10 embryos of each genotype were analyzed at each developmental stage. Embryos were fixed in $4 \%$ buffered paraformaldehyde, routinely processed, embedded in paraffin, and sectioned at 4 $\mu \mathrm{m}$. The sections were routinely stained with hematoxylin and eosin (H\&E) for qualitative and quantitative analyses.

Quantitation of kidney volume. To measure the volume of wild-type and mutant kidneys, each kidney was measured with a micrometric grid in 3 axes (longitudinal, dorsoventral, and mediolateral).

In situ hybridization. Whole-mount in situ hybridization with digoxigenin (DIG) UTP-labeled antisense riboprobes, and histological section in situ hybridization using ${ }^{35}$ S-labeled antisense riboprobes, were performed as described previously $(7,8)$. Riboprobes for $B m p 4$ were transcribed by $\mathrm{Sp} 6$ polymerase from a $1-\mathrm{kb}$ cDNA fragment (9). Alk3 (BmprIA) and Alk6 (BmprIB) riboprobes were prepared by $\mathrm{T} 7$ polymerase from $0.65-\mathrm{kb}$ and $1.5-\mathrm{kb}$ cDNA fragments, respectively (10). c-ret riboprobes were transcribed by $\mathrm{T} 7$ polymerase from the plasmid pmcret 7.2 that contains a $1.8 \mathrm{-kb}$ fragment encoding the 3 '-region of the cDNA. Wnt 11 riboprobes were prepared by $\mathrm{T} 3$ polymerase from a 2.1-kb cDNA fragment (generously provided by A.P. MacMahon, Harvard University, Cambridge, Massachusetts, USA) (11). The partial cDNA fragments for mouse gdnf and Pax2 were synthesized and amplified from embryonic kidney RNA with the following oligonucleotide: $g d n f 1,5^{\prime}$-AGAGGGAAAGGTCGCAGAGG-3', and $g d n f 2,5^{\prime}$-GGAGCCGGGGTCAGATACAT-3' (GenBank accession number, U37459); Pax2-1, 5'TGTTTCCAGCGCCTCTAACGAC-3', and Pax2-2, 5'ACTGGACTTGACTTCATCAAGCC-3' (GenBank accession number, Y07617). The amplified fragment was subcloned into PCR-II-TOPO (Invitrogen Corp., Carlsbad, California, USA), and the cRNA probes were synthesized with Sp6 polymerase.

Metanephric organ culture. Kidney rudiments were dissected from E11.5 wild-type embryos in PBS and placed on a $12-\mathrm{mm}$-diameter $0.4-\mu \mathrm{m}$ Nucleopore filter (Corning-Costar, Cambridge, Massachusetts, USA). The culture medium was DMEM supplemented with 20\% FBS (GIBCO BRL, Gaithersburg, Maryland, USA), $110 \mathrm{mg} / \mathrm{L}$ sodium pyruvate, and $0.5 \times$ streptomycin/penicillin (GIBCO BRL). To prevent spontaneous branching of the ureter, the sulfation of extracellular matrix glycosaminoglycans (GAGs) was inhibited in some cultures, by supplementing the medium with $20 \mathrm{mM} \mathrm{NaClO}$ (Sigma Chemical Co., St. Louis, Missouri, USA). In some experiments, the explants were treated with 100 $\mathrm{ng} / \mathrm{mL}$ hepatocyte growth factor (HGF) (Becton Dickinson and Co., Bedford, Massachusetts, USA), $100 \mathrm{ng} / \mathrm{mL}$ fibroblast growth factor 2 (FGF2; R\&D Systems Inc., Minneapolis, Minnesota, USA) or 100 $\mathrm{ng} / \mathrm{mL}$ BMP4. Human recombinant BMP4 was kindly provided by the Genetic Institute (Cambridge, Massachusetts, USA).
Application of exogenous protein. Affi-Gel Blue agarose beads (100-200 mesh, 75-150 $\mu \mathrm{m}$; Bio-Rad Laboratories Inc., Hercules, California, USA) were rinsed with PBS several times and soaked in $0.1 \%$ BSA or $2-5 \mu \mathrm{g} / \mathrm{mL}$ of BMP4 in $0.1 \%$ BSA in a siliconized microfuge tube for 1 hour at $37^{\circ} \mathrm{C}$. Protein-soaked beads were stored at $4^{\circ} \mathrm{C}$ for up to 1 week. Before use, all beads were washed in a drop of culture medium. The beads were then placed adjacent to the secondary branching point of E11.5 kidney rudiments, where Bmp4 is normally not expressed. In most cases, BMP4-soaked beads were placed in 1 metanephric explant, whereas control beads were placed in another explant dissected from the same embryo. The explants were then incubated with the beads for 0-72 hours and were subjected to histological analysis and whole-mount or section in situ hybridization, as indicated earlier here.

Analyses for cell death. Cell death was analyzed by the TUNEL method using the ApoTag detection kit (Intergen, Purchase, New York, USA) or Apoptosis detection kit (Promega Corp., Madison, Wisconsin, USA), according to the manufacturers' protocol.

\section{Results}

Expression of Bmp 4 and BMP type I receptor genes in the murine embryonic kidney and urinary tract system. The ontogeny of the kidney and urinary tract system begins with the budding of the ureter from the Wolffian duct (WD) toward the metanephric mesenchyme (MM). In wild-type embryos undergoing the initial ureteral budding at E10.5, Bmp4 is intensely expressed in the mesenchymal cells that surround the WD. By contrast, only a very weak Bmp4 signal is present in the WD and in the MM per se (Figure 1b), and glial cell-derived neurotrophic factor (GDNF) expression is high in the MM (data not shown). In wild-type embryos at E12.5, when the ureteric bud has undergone several rounds of branching within the kidney, Bmp4 is exclusively expressed in the stromal mesenchymal cells surrounding the main trunk and the stalk of the branching ureters that extend into the nephrogenic zone, but it is absent from the peripheral metanephrogenic mesenchyme (Figure 1d). At E14.5, in addition to the expression in the stromal mesenchymal cells, Bmp4 mRNA is seen in the Sshaped bodies and in the prospective smooth muscle layer along the ureter (Figure 1f). Two BMP type I receptor genes, Alk3 and Alk6, which encode proteins that can bind BMP4 in vitro, are also expressed during kidney and urinary tract development. Although the expression of Alk 3 is almost ubiquitous, the most intense signals are present in the WD and the branching ureter at E10.5 and E12.5, respectively (Figure 1, h and 1). Alk6 is expressed exclusively in the WD and the branching ureter at E10.5 and E12.5 (Figure 1, j and n). These expression patterns presented here are almost identical to those reported previously at E12.5 and later stages $(5,12)$, whereas there has been no reported data from E10.5. 


\section{Figure 1}

Expression of Bmp4 and BMP type 1 receptor genes during kidney and urinary tract development. Two adjacent sections from E10.5 (a, b, and $\mathbf{g}-\mathbf{j})$, E12.5 (c, d, and $\mathbf{k}-\mathbf{n})$ and E14.5 (e and $\mathbf{f}$ ) embryos were processed for (left) H\&E staining and (right) in situ hybridization of Bmp 4 (b, d, and $\mathbf{f}$ ), Alk3 (h and I), or Alk6 (j and $\mathbf{n}$ ). (a and $\mathbf{b}$ ) Bmp4 is expressed in the mesenchymal cells surrounding the WD (arrowheads), but not in MM and WD per se. (c and d) Bmp4 expression is seen around the branching ureter and ureter stalk, but not in the MM. (e and $\mathbf{f}$ ) In addition to the expression in the stromal mesenchyme, Bmp4 mRNA is distributed in the S-shaped bodies (arrowheads) and the prospective smooth muscle layer along the ureter. (g, h, $\mathbf{k}$, and $\mathbf{I})$ Expression of Alk3 is essentially ubiquitous, but noticeably intense in WD and the branching ureter (arrowheads in $\mathbf{h}$ and $\mathbf{I})$. (i, j, $\mathbf{m}$, and $\mathbf{n}$ ) Alk6 transcripts are exclusively present in the WD and the ureter epithelium (arrowheads in $\mathbf{j}$ and $\mathbf{n}$ ). WD, Wolffian duct; MM, metanephric mesenchyme; Ut, ureter; BUt, branching ureter within the kidney. Bar, $200 \mu \mathrm{m}$.

$\mathrm{Bmp}^{+/-}$mice exhibit a diverse spectrum of CAKUT. In a previous study, we showed that kidney and urinary tract anomalies are present in adult $\mathrm{Bmp}^{4^{+/-}}$mice (4). To characterize the nature of these anomalies, we first examined the morphology of the excretory system of 64 newborn (P0) $\mathrm{Bmp}^{4^{+/}}$mice and 62 wild-type mice generated by breeding of $\mathrm{Bmp}^{+/-}$males and wild-type C57BL/ 6 females. Of the $B m p 4^{+/-}$ pups, 53\% (22 males, 12 females) had grossly identifiable CAKUT, whereas no abnormal phenotype was found in wild types (Figure 2, a and e). Of those with grossly abnormal phenotypes, $47 \%$ were only on the right, $15 \%$ only on the left, and $38 \%$ bilateral.

The CAKUT found in $\mathrm{Bmp}^{+/-}$mice at $\mathrm{P} 0$ were categorized as hypo/dysplastic kidneys (HK/DKs), ureterovesical junction-type hydronephrosis (UVJ-type hydronephrosis), and duplex kidney with bifid ureters. HK/DK is characterized by a variably reduced kidney mass with microscopically dysplastic regions (Figure 2 , b and g). UVJ is accompanied by both hydronephrotic kidney and hydroureter (Figure $2 c)$. In bifid ureters, the 2 ureters from the duplex kidney unite caudally to form a single ureter that drains into the bladder with 1 ureteral orifice (Figure 2d). The prevalence of these phenotypes among $\mathrm{Bmp}^{+/-}$mice with gross anomalies was $60 \%$ $\mathrm{HK} / \mathrm{DK}, 32 \% \mathrm{UVJ}$, and $8 \%$ bifid ureters.

The histology of the affected kidneys in $B m p 4^{+/-}$ mice at birth is characterized by 2 distinct patterns. One is hydronephrosis with dilated caliceal space and thinning of renal parenchyma (Figure 2f), and the other is an abnormally small kidney with regions that are devoid of nephrogenic components, but are filled with cysts and stromal mesenchymal cells (Figure $2 \mathrm{~g}$ ), giving overall the pattern of "multicystic dysplastic kidney.”
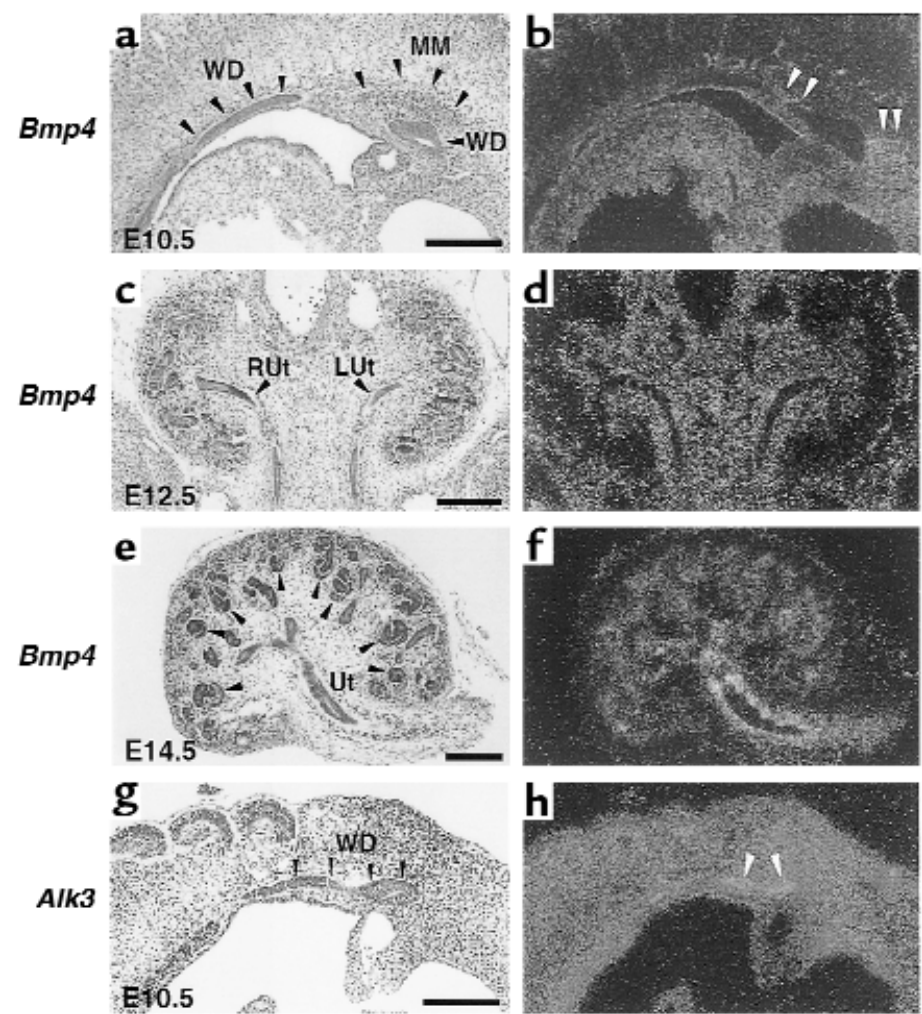

Alk6
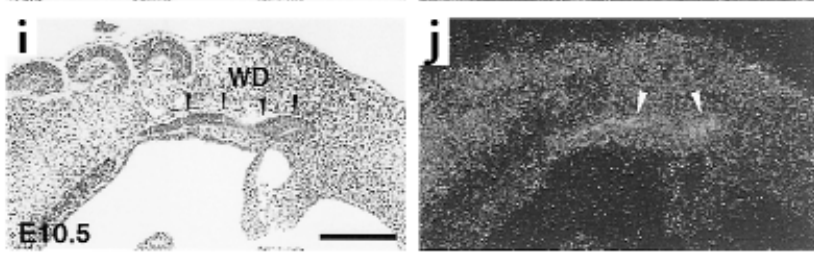

Alk3
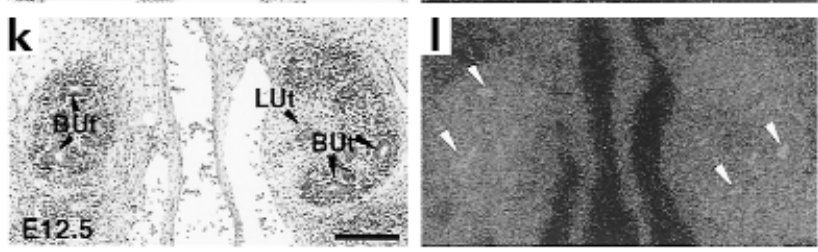

m

Alk 6
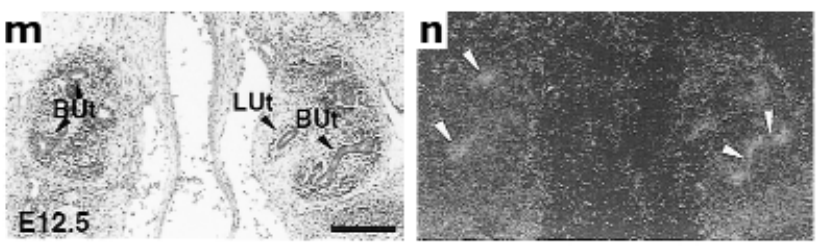

Reduced number of nephrogenic components in $\mathrm{Bmp4}^{+/-}$ embryos. In the absence of hydronephrotic change, kidneys of $\mathrm{Bmp}^{4^{+/-}}$mice are, on average, significantly smaller than those of wild-type, although the size varies among $\mathrm{Bmp4}^{+/-}$mice (Figure 2h). In utero, the difference in kidney size is already discernible by gross inspection at E14.5 (Figure 3a). Histologically, the superficial nephrogenic zone is always thinner in mutants than in wild-type, and the number of each nephrogenic component, including condensed mesenchyme and comma- and S-shaped bodies are always depressed in $\mathrm{Bmp4}^{+/-}$embryos (Figure 3c). For example, as shown in Figure 3, d and f, in E12.5 kidneys, the number of condensed mesenchyme per kidney 

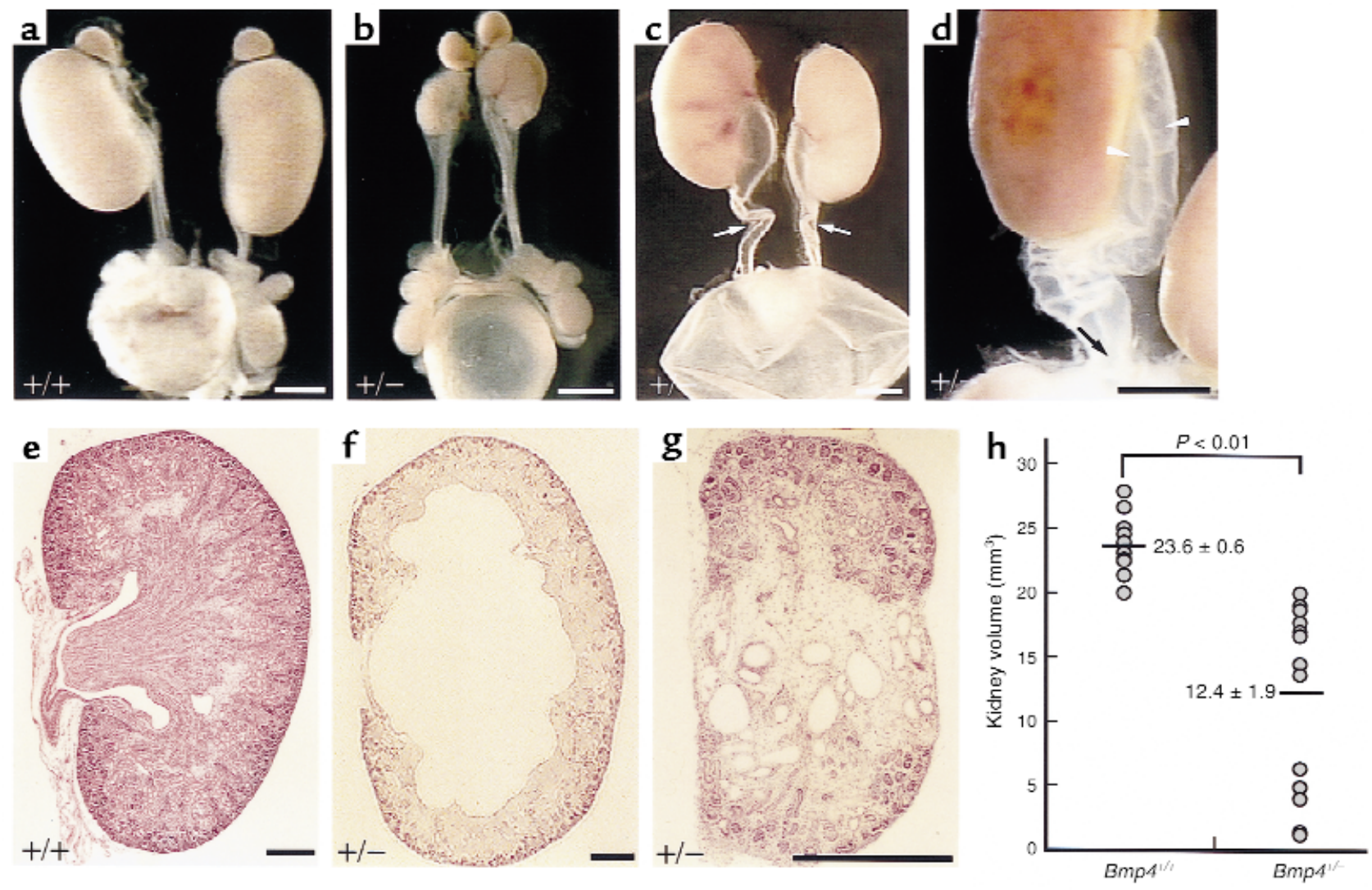

Figure 2

Gross anatomy and light microscopic histology of the congenital anomalies of kidney and urinary tract found in Bmp $4^{+/-}$pups at P0. (a-d) Gross appearance of the kidney and urinary tract system from wild-type (a) and Bmp $4^{+/-}$(b-d) mice at P0. (b) HK/DKs. (c) Hydronephrosis with hydroureter with marked tortuosity (arrows). (d) Bifid ureters, in which the 2 ureters (arrowheads) from the duplex kidney unite caudally to form a single ureter that drains into the bladder with 1 ureteral orifice. An arrow indicates the site where the 2 ureters join. (e-g) Sagittal sections of the kidneys from wild-type (e) and $\mathrm{Bmp4}^{+/-}(\mathbf{f}$ and $\mathbf{g})$ mice are shown. (f) Hydronephrosis with thinning of renal parenchyma. (g) Hypoplastic kidney (right in b) with "cystic dysplastic" regions that are devoid of nephrogenic components, but are filled with cysts and stromal mesenchymal cells. Bar, $1.2 \mathrm{~mm}(\mathbf{a}-\mathbf{d}) ; 0.4 \mathrm{~mm}(\mathbf{e}-\mathbf{g})$. (h) The volume of wild-type and heterozygous mutant kidneys was compared. The data are from 27 (wild-type, 14; $B m p 4^{+/}$, 13) kidneys of 2 litters, with obvious hydronephrotic kidneys excluded. Mean values and SEMs from each group are also shown. The difference is statistically significant by Student's $t$ test $(P<0.01)$.

is significantly lower in $\mathrm{Bmp}^{+/-}(9.0 \pm 0.88$; mean \pm SEM; $n=10)$ than in wild-type embryos $(15.6 \pm 0.51 ; n=10$; $P<0.01$ vs. $B m p 4^{+/-}$), as revealed by Pax2 whole-mount in situ hybridization. However, when kidney size is taken into account, the nephron density per se is not appreciably affected (number of condensed mesenchyme $/ \mathrm{mm}^{3}$ : for wild-type, $258.6 \pm 4.9 / \mathrm{mm}^{3}[n=10]$; for $\mathrm{Bmp}^{+/-}, 263.6$ $\left.\pm 5.9 / \mathrm{mm}^{3}[n=10]\right)$. In addition, whole-mount in situ hybridization of the E11.5 metanephros with c-ret probe showed that both the main trunk and the stems of the first 2 branches of the ureter are significantly shorter in $B m p 4^{+/-}$embryos, when compared with the wild-type at the same age (length of main ureter: for wild-type, $0.52 \pm$ $0.01 \mathrm{~mm}[n=14]$; for $B m p 4^{+/}, 0.36 \pm 0.01 \mathrm{~mm}[n=16] ; P$ $<0.001$ ) (Figure 3, e and g). Collectively, the HK/DKs are attributed to an overall slower growth and branching of the ureter in $B m p 4^{+/-}$mutants.

BMP4 promotes the growth and elongation of the ureter bud, and expands the stromal cell population of the metanephric mesenchyme. In the branching morphogenesis of the ureter, the processes of secondary budding and elongation are known to be regulated separately by distinct signaling pathways (13). Although both processes are completely inhibited when the kidney rudiments are deprived of sulfated glycosaminoglycans (S-GAGs) by treatment with chlorate ions, they are restored independently when the SGAGs-deprived kidneys are treated with exogenous factors $(14,15)$. To determine whether BMP4 can promote ureter elongation, secondary budding, or both, we have used an in vitro culture system of SGAGs-deprived kidney rudiments. When cultured in the presence of $20 \mathrm{mM}$ sodium chlorate for 72 hours, the control explants showed neither extensive growth nor ureter branching (Figure 4, a, c, and e). A BMP4loaded bead, applied adjacent to 1 of the rudimentary secondary buds, partially rescues the elongation of this bud but not the 1 farthest away (Figure 4, b, d, and f). Similar results were seen in 3 replicate experiments. Additional branching of the secondary bud was not seen, however. In addition, BMP4 promotes the overall growth of the ureter, when added directly to the cul- 


\section{Figure 3}

The ontogeny of hypo/dysplastic kidney in $B m p 4^{+/-}$embryos. (a) Gross appearance of E14.5 kidneys of wild-type (left) and heterozygous mutant (right) embryos. The mutant kidney is distinctively small. (b and c) Sagittal sections of the wild-type (b) and mutant (c) kidneys. The number of nephrogenic components per kidney is depressed in mutants. (d) Pax2 whole-mount in situ hybridization of E12.5 wild-type (left) and mutant (right) kidneys. The condensed mesenchyme is abnormally low in number in mutants. (e) c-ret whole-mount in situ hybridization of E11.5 wildtype (left) and mutant (right) kidneys. The first branch stems (arrows) and the main ureter trunk (between arrowheads) are short in mutants. The size of ureter buds is also smaller in mutants. Ut, main stem of the ureter; WD, Wolffian duct. Bar, $300 \mu \mathrm{m}$. (f) The number of condensed mesenchyme per kidney from wild-type $(n=10)$ and mutant embryos $(n$ $=10$ ) at E12.5 was quantitated. The number is significantly lower in mutant kidneys ( $P<0.01$ vs. wild-type kidneys). ( $($ ) The length of main stem of the ureter was measured in wild-type $(n=14)$ and heterozygous mutant $(n=16)$ E11.5 embryos. The difference is statistically significant by Student's $t$ test $(P<0.001)$.

ture medium with $20 \mathrm{mM}$ sodium chlorate (Figure $4 \mathrm{~g})$. This growth stimulatory effect of BMP4 is almost comparable to the known effect of HGF (Figure 4h) $(14,15)$. Thus, these in vivo and in vitro observations suggest that BMP4 regulates branching morphogenesis by promoting the growth and elongation of ureter buds once they have formed.

In addition to its effect on the ureteric bud epitheli$\mathrm{um}$, it is possible that BMP4 can regulate the development of the metanephic mesenchyme, as Alk3 is expressed not only in the epithelium, but in the metanephric mesenchyme (Figure 1, part 1). Indeed, $B m p 4^{+/-}$kidneys at E11.5 show that although both the main trunk and the stem of the first branches of the ureter are abnormally short, the secondary buds are also smaller (Figure 3e). This suggests that the process of bud formation induced by the metanephric mesenchyme can also be affected in $B m p 4^{+/-}$kidneys. In this regard, BMP7, another member of BMP superfamily, has been shown to promote the growth of the metanephric mesenchyme and the expansion of the stromal progenitor cell population in vitro in conjunction with FGF2 (16). The effect of BMP4 on the development of the metanephric mesenchyme was, therefore, examined in cultured metanephric explants $(n=$ $5)$. In the presence of FGF2, treatment of BMP4 uniformly led to an increase (1.5- to 2-fold) in size of the peripheral stromal compartment of metanephric mesenchyme, which was negative for Pax2 (Figure 5). Thus, similar to BMP7, BMP4 can stimulate the growth of the metanephric mesenchyme.

Ectopia of the ureterovesical orifice in Bmp4 $4^{+-}$mice. In addition to the hypo/dysplastic kidneys found in $B m p 4^{+-}$mice at P0, the mutant mice showed other phenotypes of CAKUT, e.g., abnormalities of the ureter. The ureters of the mutants are dilated to various degrees, with abnormal winding and kinking in the middle portion (Figure 2c). These anomalies are already present in $\mathrm{Bmp4}^{+/-}$embryos at E16.5 (Figure 6, $\mathrm{b}$ and c), but cannot be seen as early as E14.5. It has
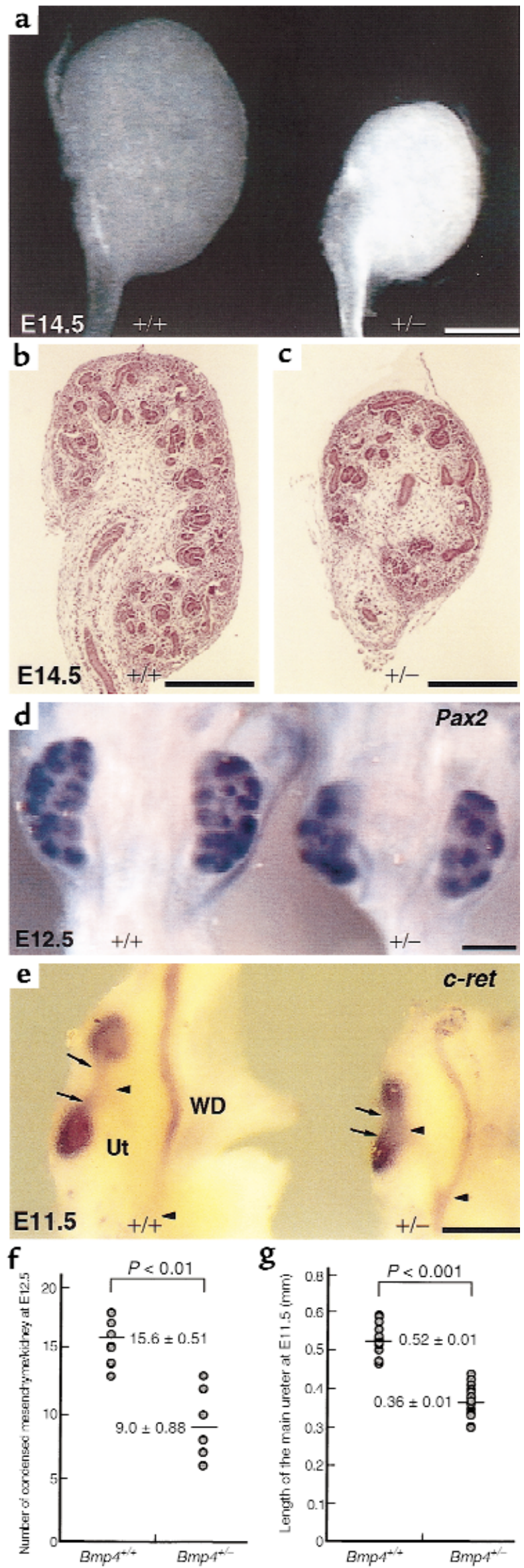


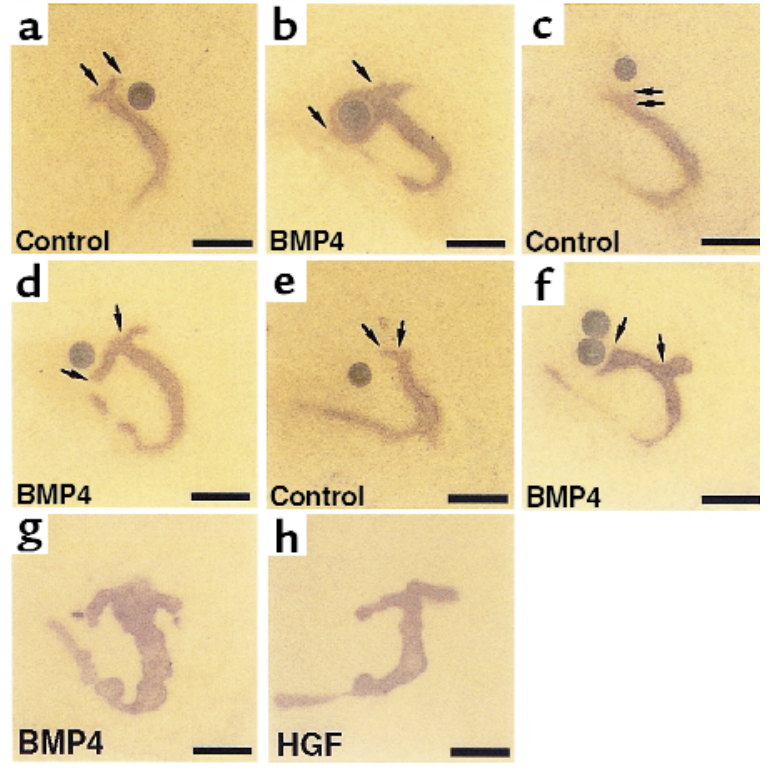

Figure 4

The effect of BMP4-loaded beads in E11.5 kidney explants deprived of S-GAGs. (a-f) BMP4-beads (b, $\mathbf{d}$, and $\mathbf{f}$ ) or control BSA beads (a, $\mathbf{c}$, and $\mathbf{e}$ ) were placed adjacent to the secondary bud of the ureter in the explants treated with $20 \mathrm{mM}$ sodium chlorate. The explants were then cultured for 72 hours and subjected to Pax2 whole-mount in situ hybridization. $\mathbf{a}$ and $\mathbf{b}, \mathbf{c}$ and $\mathbf{d}$, and $\mathbf{e}$ and $\mathbf{f}$ are pairs from 3 independent experiments. BMP4 promoted the elongation of the ureter bud adjacent to the beads (length of the ureter is indicated by the distance between arrows), but not that away from the beads. ( $g$ and $\mathbf{h}$ ) The explants were treated with $100 \mathrm{ng} / \mathrm{mL}$ of BMP4 (g) or HGF (h) for 72 hours in the presence of $20 \mathrm{mM}$ sodium chlorate. The growth stimulatory effect was comparable between BMP4 and HGF. Bar, $300 \mu \mathrm{m}$.

been demonstrated by extensive scrutiny of human specimens that ectopia of the ureterovesical (UV) orifice is central to CAKUT, as it causes or accompanies anomalies of the ureter, including UVJ obstruction, vesicoureteral reflux, megaureter, and hypodysplasia of nephrons (17). We therefore examined whether ectopic UVJ site occurs in $\mathrm{Bmp}^{+/-}$embryos. Inspection of the UV orifice inside the bladder by dissection revealed that the site of the orifice is abnormally close to the urethral orifice and that the distance between right and left ureteral orifices is appreciably shorter in $B \mathrm{mp} 4^{+/}$ embryos than in wild-type embryos at E16.5 (Figure $6 \mathrm{e})$. The latter finding is further confirmed by histological analysis of tissue sections (Figure $6 \mathrm{~g}$ ). The same abnormality is also present in 11 of the 20 mutant pups studied at birth. In the most severely affected mutants, the ureter failed to connect to the bladder, but instead connected aberrantly to the seminal vesicle or vas deferens, both of which are WD derivatives (Figure 6, h and i). Thus, the UV orifice of $B m p 4^{+/-}$mice lies abnormally caudal to its normal location.

Ectopia of the UV orifice results from aberrant initial ureteral budding in $\mathrm{Bmp}^{+/-}$mutants. During embryonic rearrangement of the urinary tract, the UV orifice migrates from the initial budding site on the WD to the cloaca. The final position of the UV orifice therefore reflects the site of initial ureteral budding and subsequent complex processes, including the absorption into the cloaca of the portion of WD between the ureteric bud and the cloaca, known as the common mesonephric duct (CMD) (18). To examine the ontogeny of the ectopic UV orifice in $B m p 4^{+/-}$mutants, we traced the UVJ site back into early embryos. At E13.5, the ureter is already connected to the cloaca in wild-type embryos (Figure 7a), whereas the ureter of the mutants still drains into the WD (Figure 7b). At E12.5, when the ureter still comes off the WD even in wild-type embryos (Figure 7c), the length of the CMD is abnormally long in $B m p 4^{+/-}$embryos when compared with wild-type (Figure 7d). At this stage, the CMD is actively absorbed into the cloaca in wild-type embryos through, at least in part, extensive apoptosis, as evidenced by numerous TUNEL-positive nuclei highly localized in the epithelium of the CMD (Figure 7e). The longer-than-normal $\mathrm{CMD}$ of $\mathrm{Bmp}^{+/-}$embryo also has the same density of TUNEL-positive cells as does the wild type (Figure 7f), indicating that the absorption of the CMD through apoptosis is comparable between genotypes. We therefore investigated the possibility that the CMD of $\mathrm{Bmp}^{+/-}$embryos is already longer than normal at the time of initial ureteral budding. For this purpose, we identified the site of the initial ureteral budding at E11.0 by c-ret whole-mount in situ hybridization. Simultaneously, we used a Wnt 11 probe, as Wnt 11 is expressed in all of the somite pairs at this stage. Although Wnt 11 is also expressed in the ureteric bud epithelium at this stage, the initial budding site cannot be clearly identified with the Wnt 11 probe alone. When comparing mutant $(n=5)$ and wild-type $(n=4)$ embryos with an identical number of somite pairs (41), the position of the primary bud in the wild-type is opposite the approximately 26th somite, whereas in 3 mutants it is opposite the approximately 25 th somite (Figure $7, \mathrm{~g}$ and $\mathrm{h}$ ). Thus, the more caudal location of the final UV orifice
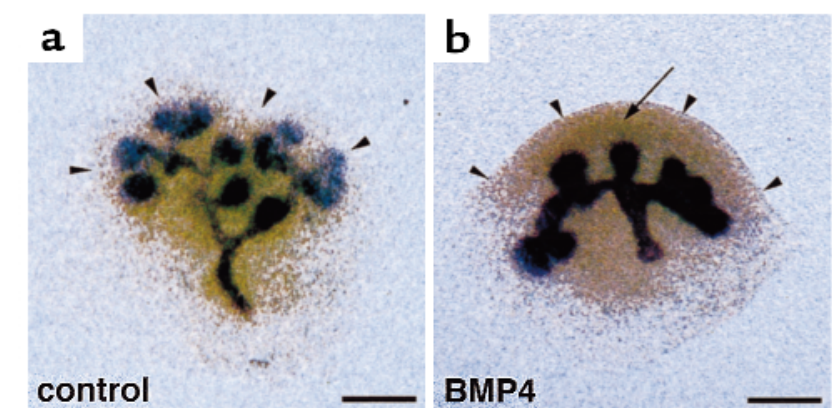

\section{Figure 5}

The effect of exogenous BMP4 on the growth of metanephric mesenchyme in cultured metanephric explants. The E11.5 kidney rudiments were incubated with BMP4 (b) or vehicle (a) in the presence of FGF2 for 48 hours and subjected to a Pax2 whole-mount in situ hybridization. BMP4 promotes an expansion of the Pax2-negative peripheral stromal compartment of the metanephric mesenchyme (arrow). Arrowheads indicate an edge of the explants. Bar, $300 \mu \mathrm{m}$. 
in $\mathrm{Bmp}^{+/-}$mice may result from the more cranial position of the initial ureteral buds along the WD.

The ectopic budding from the main trunk of the ureter of $B m p 4^{+/-}$embryos. In addition to the phenotypes already described here, we found $4 \mathrm{Bmp}^{+/-}$newborns that have another distinctive CAKUT phenotype, i.e., bifid ureters (Figure $2 \mathrm{~d}$ ). In all of these 4 mutants, the 2 ureters from the duplex kidney join caudally to form a common stem outside the bladder wall, which drains into the bladder through 1 ureteral orifice. A duplex collecting system is thought to be the result of extra budding of the ureter during early stages of kidney and urinary tract development (18). Although no accessory budding from the WD is seen in $B m p 4^{+/-}$embryos at E10.5, of 19 mutants at E11.5, 2 had accessory buds from the main stem of the ureter, where budding is normally never seen (Figure 8, a and b).

One essential regulator of ureter budding is GDNF, which acts on the ureter through its receptor, c-ret. GDNF regulates the branching morphogenesis of the ureter by stimulating bud initiation and by determining bud orientation $(13,15,19)$. The ectopic budding found in $\mathrm{Bmp}^{+/-}$embryos raises the possibility that BMP4 modulates GDNF action on the stalk of the branching ureter, where $B m p 4$ is intensely expressed in the surrounding mesenchyme (Figure 1d). To test this possibility, we examined, in the explant culture system, the effect of BMP4 on the expression of Wnt 11, which is a molecular marker for ureter buds and whose expression is rapidly regulated by GDNF signaling (20, 21). When BMP4-loaded beads were placed adjacent to

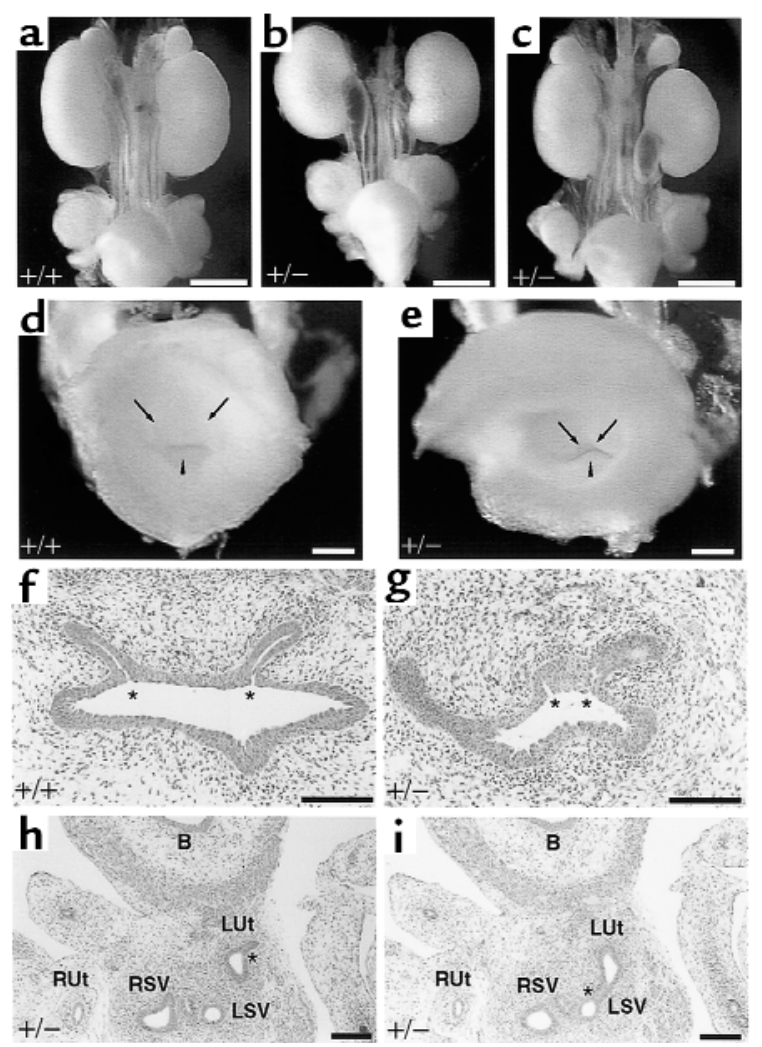

The Journal of Clinical Investigation

\begin{tabular}{l|l|l} 
April $2000 \quad$ Volume $105 \quad$ Number 7
\end{tabular} the secondary branching point of E11.5 metanephric explants, a position where Bmp4 is not normally expressed, branching is markedly inhibited in the area surrounding the bead after 48 hours and 72 hours of culture (Figure 9, b and d). In addition, BMP4-beads downregulated the expression of Wnt 11 in the adjacent branching ureter after a culture period of 8 hours (Figure 9f) and almost abolished its expression after 16 hours of incubation (Figure 9g). This was further confirmed by histological section in situ hybridization that showed that the ureter epithelium adjacent to BMP4-beads lost Wnt 11 expression (data not shown). By contrast, the expression level of GDNF was not appreciably downregulated in the areas surrounding BMP4-loaded beads when compared with control beads during a culture period of 16 hours (Figure 9, i-1). Thus, BMP4 can inhibit the effect of GDNF signaling (Wnt 11 expression) in the ureter epithelium without directly modulating GDNF expression per se.

\section{Discussion}

In the present study, we have examined in detail the nature of the kidney and urinary tract anomalies of $\mathrm{Bmp}^{+/-}$mice. Although $\mathrm{Bmp}^{+/-}$mice have abnormal phenotypes in other organs (e.g., polydactyly, microphthalmia, craniofacial defects, and abnormalities in male accessory glands), their overall growth and embryonic development (assessed by body weight, crown-rumps length, and the number of somites) and litter size are not significantly different from wild-type.

Our study began with the morphological analysis of the kidney and urinary tract immediately after birth. This revealed that $B m p 4^{+/-}$mutants have diverse CAKUT, including HK/DK, hydronephrosis with hydroureter, and duplex kidney/ureter system. These murine CAKUT mimic human CAKUT in several ways. Thus, in $B m p 4^{+/-}$mutants, a wide anatomical spectrum was seen within 1 litter, with each specific anatomical pattern having its counterpart in human (22). As in humans, the murine CAKUT appear predominantly in males (23-25). In addition, human CAKUT often show a familial pattern with incomplete and variable penetrance, as in the murine

\section{Figure 6}

Ectopia of UV orifice in Bmp4 $4^{+/-}$mutants. All the panels are from E16.5 wild-type (a, d, and $\mathbf{f}$ ) and heterozygous mutant (b, c, e, and $\mathbf{g - i}$ ) embryos. (a-c) Gross morphology of the urogenital system is shown. Hydroureter is already present in E16.5 mutant embryos (b and c). (d and $\mathbf{e}$ ) Macroscopic appearance of the UV orifice inside the bladder. The position of the UV orifice (arrows) is abnormally close to the orifice of the urethra (arrowheads) in the mutant (e). ( $\mathbf{f}$ and $\mathbf{g}$ ) Microscopic appearance of the UV orifice. The distance between the left and right orifices (asterisk) is short in the mutant ( $\mathbf{g})$, when compared with the wild-type (f). (h and $\mathbf{i}$ ) Adjacent transverse sections of the urogenital tract from 1 mutant embryo. The left ureter changes direction posteriorly at the site indicated by the asterisk in $\mathbf{h}$ and connects to the left seminar vesicle, as indicated by the asterisk in $\mathbf{i}$. B, bladder; Ut, ureter; SV, seminal vesicle. L, left; R, right. Bar, $1 \mathrm{~mm}(\mathbf{a}-\mathbf{c}) ; 200 \mu \mathrm{m}$ (d and $\mathbf{e}) ; 100 \mu \mathrm{m}(\mathbf{f}-\mathbf{i})$. 
CAKUT (26). Finally, mice and humans also share the same renal histological characteristics, namely lack of interstitial fibrosis, with some hypoplastic and cystic dysplastic parenchyma. The penetrance of CAKUT in $\mathrm{Bmp4}^{+/-}$mutants is high (Figures $2 \mathrm{~h}$ and $3 \mathrm{f}$ ), and the origin of CAKUT can be traced back to early embryogenesis.
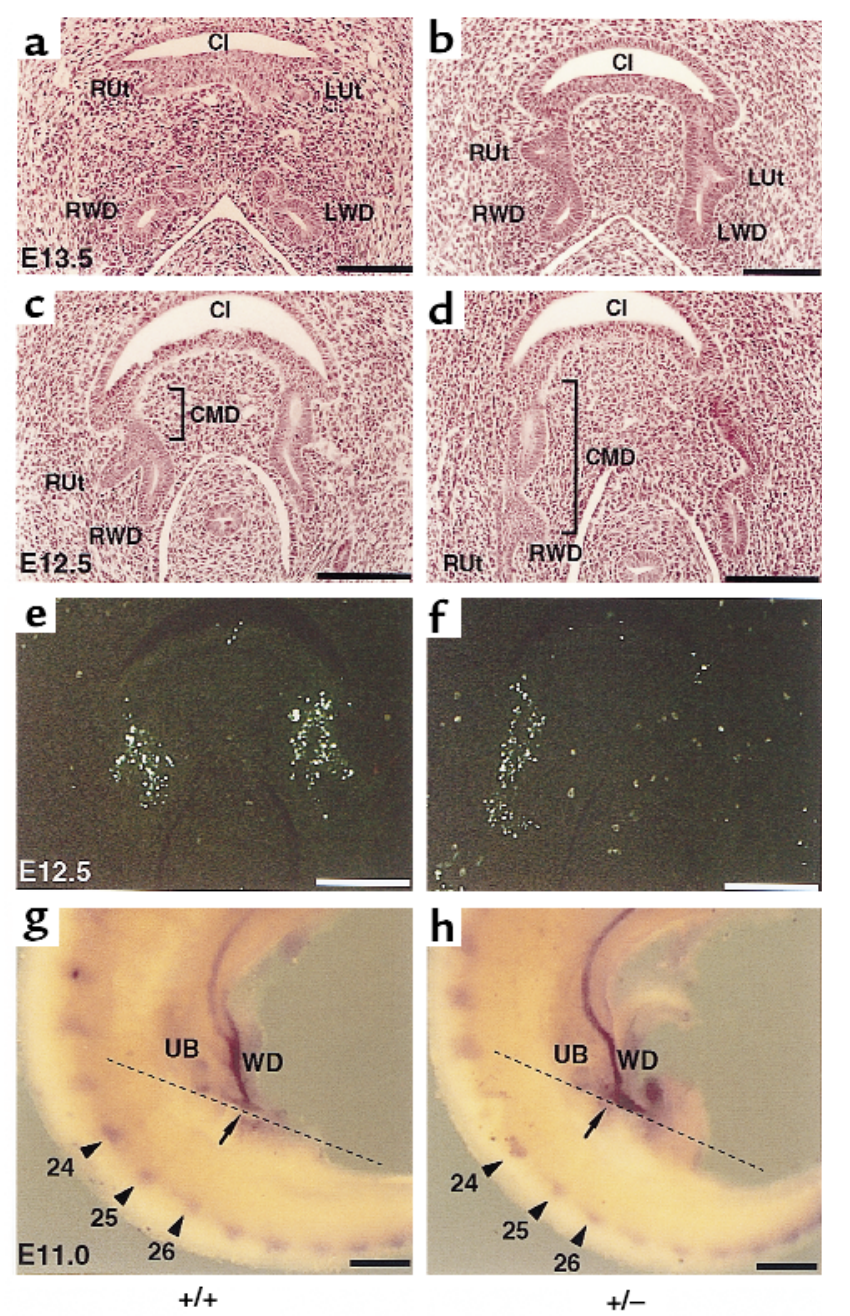

\section{Figure 7}

Ontogeny of ectopic UV orifice in $B m p 4^{+/-}$embryos. (a and b) At E13.5, the ureter is connected to the cloaca in the wild type (a), whereas the ureter of the mutant is still connected to WD (b). (c and d) At E12.5, the ureter still drains to WD in both the wild type (c) and the mutant (d). The length of the CMD is abnormally long in the mutant. $\mathbf{e}$ and $\mathbf{f}$ are adjacent sections to $\mathbf{c}$ and $\mathbf{d}$, respectively, and subjected to TUNEL staining. Apoptosis of CMD is comparable between the wild type (e) and mutant $(\mathbf{f})$. ( $\mathbf{g}$ and $\mathbf{h}) \mathrm{c}$-ret and Wnt 11 whole-mount in situ hybridization for wild-type $(\mathbf{g})(n=4)$ and mutant (h) $(n=3)$ E11.0 embryos. The position of the budding from WD is indicated by arrows, and the 24th, 25th, and 26th somite pairs are marked by arrowheads. The position of the budding in the wild type corresponds to the approximately 26 th somite (g), whereas that in the mutant corresponds to the approximately 25 th somite (h). The dashed lines in $\mathbf{g}$ and $\mathbf{h}$ show the approximate plane of the sections for a-f. Ut, ureter; WD, Wolffian duct; CMD, common mesonephric duct; $\mathrm{Cl}$, cloaca; UB, ureteric bud.L, left; R, right. Bar, $100 \mu \mathrm{m}(\mathbf{a}-\mathbf{f}) ; 300 \mu \mathrm{m}$ (g and $\mathbf{h})$.
Although a large variation exists in kidney size of $B \mathrm{mp} 4^{+/-}$mice (Figure $2 \mathrm{~h}$ ), almost all of the mutant kidneys contain cystic dysplastic regions to a various degree. In utero, the number of nephrogenic components at various developmental stages is reduced, although the nephron density is preserved and tubulogenesis remains intact, as evidenced by histological and whole-mount analysis of the mutant kidneys. In addition, at E11.5, the size of the T-shaped bud (the main ureter trunk, the first branch stem, and the secondary buds) is abnormally small in mutants, when compared with the wild type. Thus, the HK/DK of $\mathrm{Bmp4}^{+/-}$ mutants is attributed to a reduced growth and branching of the ureter, which can lead to low nephron number by birth (27).

Branching of the ureter can be divided into 3 repetitive stages, namely, bud initiation, bud orientation, and bud elongation (13). We have shown, in the culture system of metanephric explants deprived of SGAGs, that BMP4 does not increase the number of branches, but partially rescues the growth and elongation of ureter buds in a manner similar to HGF (14, 15). This observation is consistent with the expression patterns of Bmp4 and BMP type I receptor genes. Thus, although Bmp4 mRNA is exclusively expressed in the mesenchymal cells surrounding the elongating stalk of the ureter, and not around the tip of the branching ureter, the type I receptor genes are expressed in the ureter epithelium. The observed stimulatory effect of BMP4 on the outgrowth of the ureter is similar to that of FGF7, which is required for the growth of the ureteric bud both in vivo and in vitro (28). However, FGF7 mRNA is not detected in the urogenital system until E14.5, whereas Bmp4 is intensely expressed as early as E10.5, suggesting that BMP4 appears to be important for bud growth and elongation particularly during the early stage of the kidney development. It should be noted that the final length of the main ureter may not be short in $B m p 4^{+/-}$mice at birth (Figure 2c) owing to secondary changes, e.g., obstructive uropathy.

As the abnormally small secondary buds (Figure 3e) and the dysplastic morphology (Figure $2 \mathrm{~g}$ ) in $\mathrm{Bmp4}^{+/-}$ kidneys at E11.5 and at birth, respectively, cannot be explained only as a result of incomplete elongation of the branching ureters, it is plausible that BMP4 can also regulate the development of the metanephric mesenchyme. In this regard, it has been demonstrated in the mutant embryos carrying inactivated winged-helix transcription factor BF2 (29) or retinoic acid receptor (RAR) gene (30) that the stromal compartment of metanephric mesenchyme is essential for the kidney morphogenesis and ureteric bud branching. We have shown that, similar to BMP7, exogenous BMP4 increases the size of Pax2-negative peripheral stromal cell population in the presence of FGF2 in cultured metanephric explants (Figure 5). Because BMP2 and BMP7, which are known to share common biologic functions with BMP4 (1), can act as a survival 
Figure 8

Ectopic budding from the main stem of the ureter in E11.5 mutant embryos. c-ret whole-mount in situ hybridization for wild-type (a) and mutant (b) kidneys. An arrow in $\mathbf{b}$ indicates the ectopic budding from the main trunk of the ureter (between arrowheads) in the mutant, whereas budding is never seen in wild-type embryos (a). Ut, ureter; WD, Wolffian duct. Bar, $100 \mu \mathrm{m}$.

factor for the isolated metanephric mesenchyme (16, 31 ), it is conceivable that BMP4 may stimulate proliferation and/or prevent cell death of the metanephric mesenchyme, in addition to the direct effects on the epithelium (Figure 10).

In addition to its growth-promoting function, BMP4 has another effect on the branching ureter, i.e., inhibition of ectopic budding in the stalk of the ureter. Although the incidence is low, of $64 \mathrm{Bmp}^{+/-}$ newborn mice, 4 were found to have a distinctive phenotype of duplex kidney with bifid ureters. The duplex collecting system is thought to be attributed to additional ureteral budding from an aberrant position in the WD or the ureter during early stages of kidney and urinary tract development (18). Indeed, in

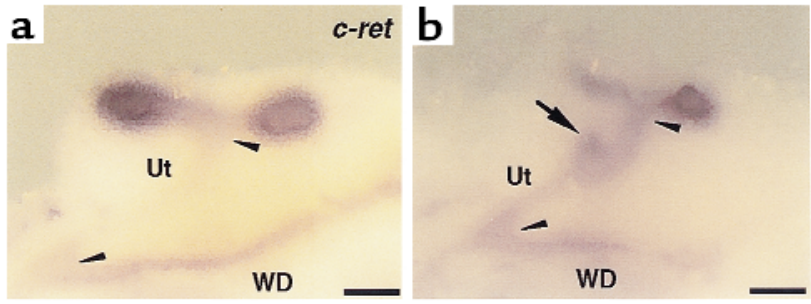

2 mutant embryos at E11.5, we could capture the initial process of extra budding from the main stem of the ureter, a site where buds normally never emerge. The mechanism for limiting the site of the budding to the tip of the branching ureter in wild-type animals has heretofore been largely unknown. Although the tightly regulated expression pattern of c-ret and its ligand GDNF may be part of the mechanism to specify the site of branching, both c-ret and GDNF are expressed surprisingly broadly throughout the branching ureter and metanephric mesenchyme, respectively, at early stage of kidney development (E10.5-E12.0) (Figures 3e, 8, and 9, i-l) (32). From the expression pattern of $B m p 4$ and the presence of the bifid ureters resulting from the ectopic budding in

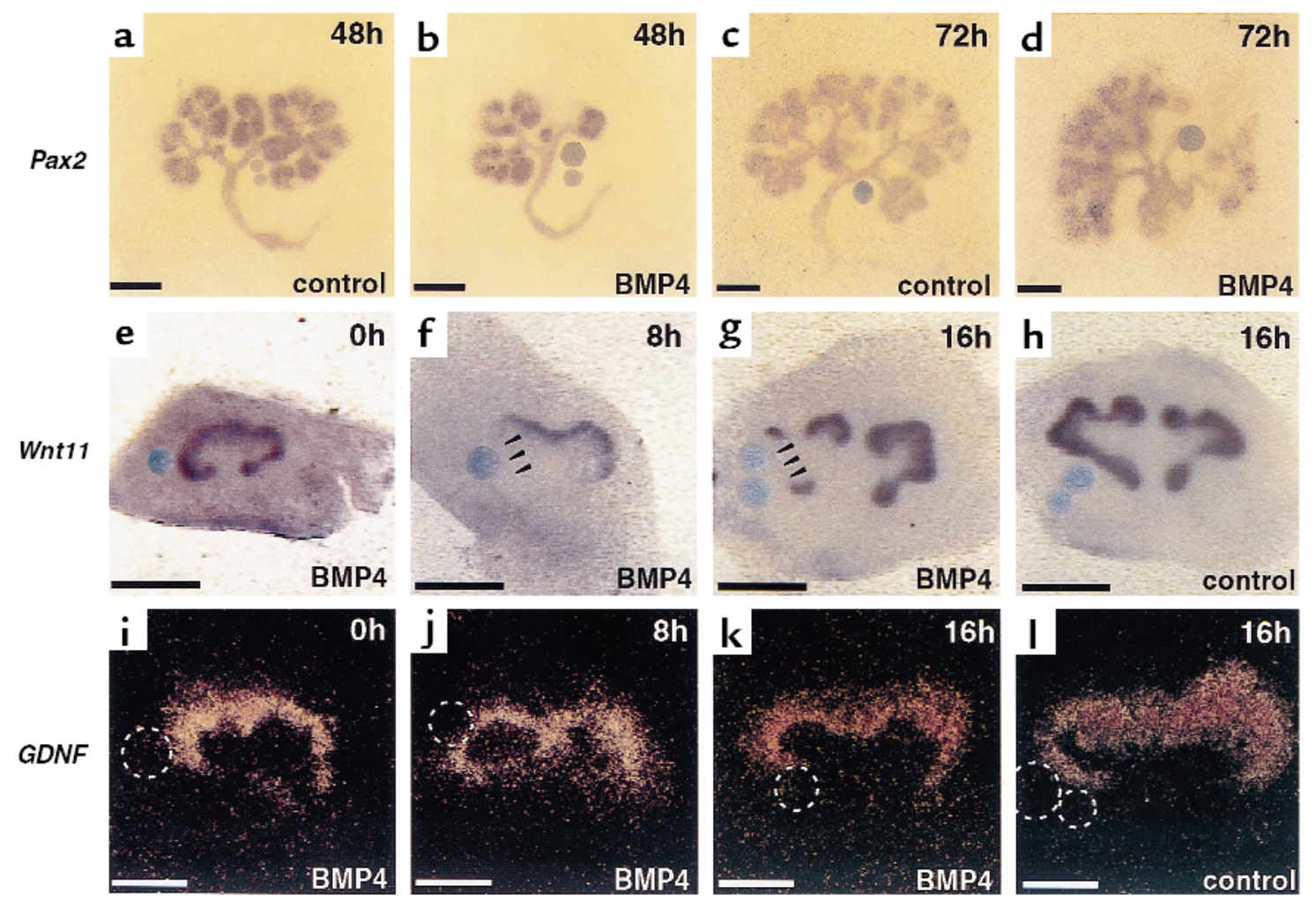

Figure 9

BMP4 downregulates the expression of Wnt 11 in metanephric explants. (a-d) The explants are incubated for 48 hours (a and b) or 72 hours ( $\mathbf{c}$ and $\mathbf{d}$ ) with BMP4 beads ( $\mathbf{b}$ and $\mathbf{d}$ ) or control BSA beads ( $\mathbf{a}$ and $\mathbf{c}$ ) that have been placed adjacent to the secondary buds and subjected to Pax2 whole-mount in situ hybridization. Branching is inhibited in the area surrounding the bead. (e-h) Wnt 11 expression in the explants incubated with BMP4 beads for 0 hours (e), 8 hours (f), and 16 hours (g) or control beads for 16 hours (h). Wnt 11 expression almost disappeared at the ureter epithelium around the BMP4 beads, as indicated by arrowheads. (i-I) Expression of GDNF in the explants treated with BMP4-beads for 0 hours (i), 8 hours $(\mathbf{j})$, and 16 hours $(\mathbf{k})$ or control beads for 16 hours $(\mathbf{I})$. GDNF expression is almost comparable between the areas surrounding BSA- and BMP4-soaked beads. Dashed circles indicate the location of the beads. Bar, $300 \mu \mathrm{m}$. 


\section{Figure 10}

A model for the possible roles of BMP4 during branching morphogenesis of the ureter. BMP4 expressed in the mesenchyme acts through BMP type I receptors (ALK3 and ALK6) in the Wolffian duct and the stalk of branching ureter to promote bud growth and elongation and inhibits ectopic budding by antagonizing GDNF action. In addition, BMP4 may promote the growth of metanephric mesenchyme through ALK3 by regulating proliferation and/or apoptosis of these cells, which produces a factor(s) to stimulate bud formation.

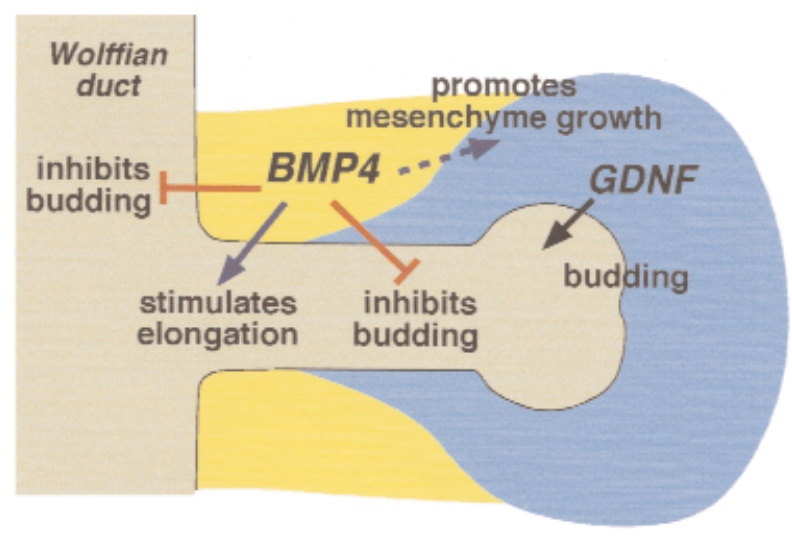

bud formation on the WD has not been fully understood. Whereas GDNF mRNA is broadly distributed in the posterior region of the WD, and c-ret mRNA is expressed throughout the WD in wild-type embryos undergoing initial ureteral budding, the actual budding occurs from a finely restricted region of the WD and penetrates the caudal end of the MM. Assuming that the function of the Bmp4 expressed around the WD is analogous to that expressed within the kidney, BMP4 may play a role in specifying the site of the initial ureteral budding by antagonizing GDNF signaling on the WD.

In summary, during kidney and urinary tract development, Bmp4 is exclusively expressed in the stromal mesenchymal cells that surround WD and the stalk of the ureteric bud, but not in the MM that surrounds the tip of the ureteric bud, whereas BMP type I receptors (Alk3/6) are expressed highly or exclusively, respectively, in WD and the ureter epithelium. We propose a model in which BMP4, acting on its receptors along the epithelium, determines the site of initial ureteral budding on the WD and regulates the branching morphogenesis of the ureter by antagonizing GDNF signaling and promoting the growth and elongation of the ureter. In addition, BMP4 may stimulate the growth of metanephric mesenchyme, which indirectly produces factors to promote branching morphogenesis of the ureter (Figure 10). A defect in these functions can lead to development of CAKUT in $\mathrm{Bmp4}^{+/-}$mice that anatomically and histologically mimic human CAKUT, with a diverse spectrum of anomalies including HK/DK, urinary tract anomalies along with ectopia of the ureteral orifice, and double collecting system.

\section{Acknowledgments}

The authors thank T. Kume, R. Dunn, Y. Furuta, and M. Weaver for technical advice; T. Bills for technical assistance; and J.C. Pope for helpful comments. This work was supported by grants from the National Institute of Health (DK-44757 and DK-37868). B.L.M. Hogan is an Investigator of the Howard Hughes Medical Institute. A. Fogo is a recipient of an Established Investigator Award from the American Heart Association. 
1. Hogan, B.L.M. 1996. Bone morphogenetic proteins: multifunctional regulators of vertebrate development. Genes Dev. 10:1580-1594.

2. Winnier, G., Blessing, M., Labosky, P.A., and Hogan, B.L.M. 1995. Bone morphogenetic protein- 4 is required for mesodermal formation and patterning in the mouse. Genes Dev. 9:2105-2116.

3. Lawson, K.A., et al. 1999. Bmp4 is required for the generation of primordial germ cells in the mouse embryo. Genes Dev. 13:424-436.

4. Dunn, N.R., et al. 1997. Haploinsufficient phenotypes in Bmp4 heterozygous null mice and modification by mutations in Gli3 and Alx 4 . Dev. Biol. 188:235-247.

5. Dudley, A.T., and Robertson, E.J. 1997. Overlapping expression domains of bone morphogenetic protein family members potentially account for limited tissue defects in BMP7 deficient embryos. Dev. Dyn 208:349-362.

6. Pope, J.C., Brock, J.W., Adams, M.C., Stephens, F.D., and Ichikawa, I. 1999. How they begin and how they end: classic and new theories for the development and deterioration of congenital anomalies of the kidney and urinary tract, CAKUT. J. Am. Soc. Nephrol. 10:2018-2028.

7. Hogan, B.L.M., Beddington, R., Constantini, F., and Lacy, E. 1994. Manipulating the mouse embryo. A laboratory manual. Cold Spring Harbor Laboratory Press. Plainview, NY. 352 pp.

8. Kakinuma, Y., Fogo, A., Inagami, T., and Ichikawa, I. 1993. Intrarenal localization of angiotensin II type 1 receptor mRNA in the rat. Kidney Int. 43:1229-1235.

9. Jones, C.M., Lyons, K.M., and Hogan, B.L.M. 1991. Involvement of bone morphogenetic protein-4 (BMP-4) and Vgr-1 in morphogenesis and neurogenesis in the mouse. Development. 111:531-542.

10. Furuta, Y., and Hogan, B.L.M. 1998. BMP4 is essential for lens induction in the mouse embryo. Genes Dev. 12:3764-3775.

11. Kispert, A., Vainio, S., Shen, L., Rowitch, D.H., and McMahon, A.P. 1996. Proteoglycans are required for maintenance of Wnt11 expression in the ureter tips. Development. 122:3627-3637.

12. Dewulf, N., et al. 1995. Distinct spatial and temporal expression patterns of two type I receptors for bone morphogenetic proteins during mouse embryogenesis. Endocrinology. 136:2652-2663.

13. Sariola, H., and Sainio, K. 1997. The tip-top branching ureter. Curr. Opin Cell Biol. 9:877-884.

14. Davies, J., Lyon, M., Gallagher, J., and Garrod, D. 1995. Sulfated proteoglycan is required for collecting duct growth and branching but not nephron formation during kidney development. Development. 121:1507-1517.

15. Sainio, K., et al. 1997. Glial-cell-derived neurotrophic factor is required for bud initiation from ureteric epithelium. Development. 124:4077-4087.

16. Dudley, A.T., Godin, R.E., and Robertson, E.J. 1999. Interaction between FGF and BMP signaling pathways regulates development of metanephric mesenchyme. Genes Dev. 13:1601-1613

17. Mackie, G.G., and Stephens, F.D. 1975. Duplex kidneys: a correlation of renal dysplasia with position of the ureteral orifice. J. Urol. 114:274-280.
18. Stephens, F.D., and Huston, J.M. 1996. Congenital anomalies of the urinary and genital tracts. ISIS Medical Media. Oxford, United Kingdom. 472 pP.

19. Tang, M.-J., Worley, D., Sanicola, M., and Dressler, G.R. 1998. The RETglial cell-derived neurotrophic factor (GDNF) pathway stimulates migration and chemoattraction of epithelial cells. J. Cell Biol. 142:1337-1435.

20. Pepicelli, C.V., Kispert, A., Rowitch, D.H., and McMahon, A.P. 1997. GDNF induces branching and increased cell proliferation in the ureter of the mouse. Dev. Biol. 192:193-198.

21. Ehrenfels, C.W., Carmillo, P.J., Orozco, O., Cate, R.L., and Sanicola, M. 1999. Perturbation of RET signaling in the embryonic kidney. Dev. Genet. 24:263-272

22. Brown, T., Mandell, J., and Lebowitz, R.L. 1987. Neonatal hydronephrosis in the era of sonography. Am. J. Roentgenol. 148:959-963.

23. Johnston, J.H., Evans, J.P., Glassberg, K.I., and Shapiro, S.R. 1977. Pelvic hydronephrosis in children: a review of 219 personal cases. J. Urol. 117:97-101.

24. Najmaldin, A., Burge, D.M., and Atwell, J.D. 1990. Reflux nephropathy secondary to intrauterine vesicoureteric reflux. J. Pediatr. Surg. 25:387-390.

25. Coret, A., et al. 1994. The impact of fetal screening on indications for cystourethrography in infants. Pediatr. Radiol. 24:516-518.

26. Larson, R.S., et al. 1995. The Ivemark syndrome: prenatal diagnosis of an uncommon cystic renal lesion with heterogeneous associations. Pediatr. Nephrol. 9:594-598

27. Oliver, J. 1968. Nephrons and kidneys: a quantitative study of developmental and evolutionary renal architecture. Hoeber Medical Division, Harper and Row. New York, NY. 35-38.

28. Qiao, J., et al. 1999. FGF-7 modulates ureteric bud growth and nephron number in the developing kidney. Development. 126:547-554.

29. Hatini, V., Huh, S.O., Herzlinger, D., Soares, V.C., and Lai, E. 1996. Essential role of stromal mesenchyme in kidney morphogenesis revealed by targeted disruption of Winged Helix transcription factor BF-2. Genes Dev. 10:1467-1478.

30. Mendelsohn, C., Batourina, E., Fung, S., Gilbert, T., and Dodd, J. 1999. Stromal cells mediate retinoid-dependent functions essential for renal development. Development. 126:1139-1148.

31. Godin, R.E., Takaesu, N.T., Robertson, E.J., and Dudley, A.T. 1998. Regulation of BMP7 expression during kidney development. Development. 125:3473-3482.

32. Srinivas, S., Wu, Z., Chen, C.-M., D’Agati, V., and Constantini, F. 1999. Dominant effects of RET receptor misexpression and ligand-independent RET signaling on ureteric bud development. Development. 126:1375-1386.

33. Piscione, T.D., et al. BMP-2 and OP-1 exert direct and opposite effects on renal branching morphogenesis. Am. J. Physiol. 273: F961-F975.

34. Neubuser, A., Peters, H., Balling, R., and Martin, G.R. 1997. Antagonistic interactions between FGF and BMP signaling pathway: a mechanism for positioning the sites of tooth formation. Cell. 90:247-245. 